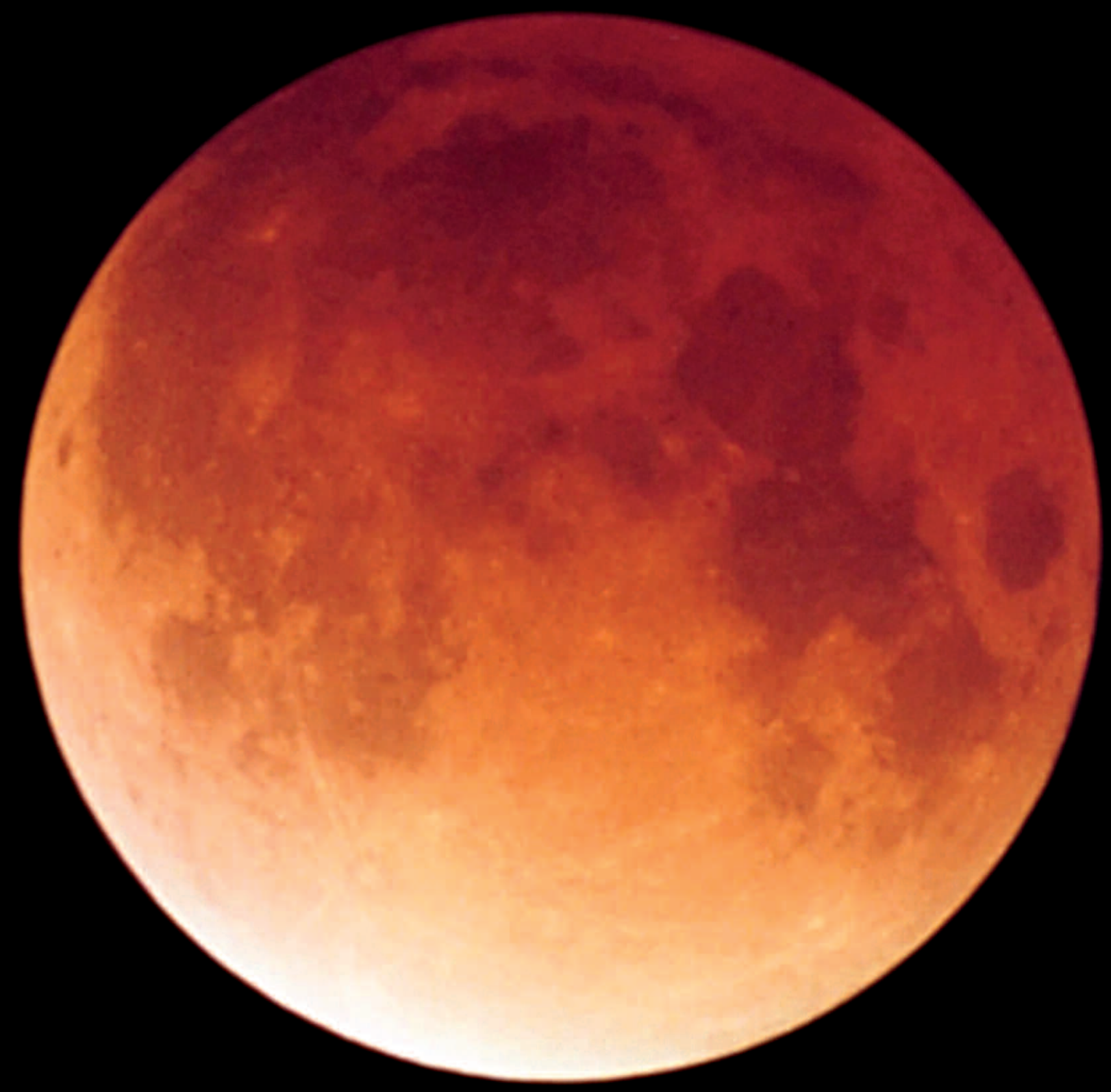

\title{
The moon as a detector of Ultra-High-Energy neutrinos
}

口 Olaf Scholten - KVI, University of Groningen - Groningen, The Netherlands - DOI: 10.1051/epn/2011506 


\section{When ultra-high-energy (UHE) particles impact on the lunar surface they initiate a particle cascade. The sizeable negative charge excess will cause emission of coherent radio waves, making these impacts detectable on Earth with sensitive radio telescopes. Using the Westerbork Synthesis Radio Telescope array we searched for these impacts to uncover UHE particle origins.}

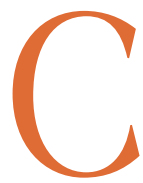

osmic rays and neutrinos at ultra-high energies are the messengers of the most energetic processes in the universe. The particles have energies well in excess of $10^{20} \mathrm{eV}$, far above energies that are reachable at CERN and almost equal to the kinetic energy of $1 \mathrm{~kg}$ falling from a height of $2 \mathrm{~m}$. At energies E below $0.5 \times 10^{20} \mathrm{eV}$ their flux [1] falls roughly as $\mathrm{E}^{-2.7}$. It is generally assumed that the particles are accelerated to these extreme energies by the so-called Fermi acceleration mechanism, where the average particle energy increases by repeated interactions with a shock wave [2]. This mechanism produces a power-law spectrum as is observed. In alternative top-down models, the high energy particles result from the decay of massive particles that are relics from the Big Bang. The UHE sources of origin are not known, although close to $20 \%$ of the UHE particles seem to come from the direction of Centaurus A [3]. This is a bright radio galaxy in the southern hemisphere constellation Centaurus, emitting copious amounts of synchrotron radiation. At a distance of only $11 \mathrm{mil}-$ lion light-years it is the closest such galaxy. A major challenge in finding the sources of origin is the very small observed flux, of the order of a single particle per $100 \mathrm{~km}^{2}$ per year for energies in excess of $10^{20} \mathrm{eV}$. At higher energies the flux of cosmic rays falls with energy even faster than $\mathrm{E}^{-2.7}$, perhaps because of the so-called GZK effect. Greisen, Zatsepin and Kuzmin predict that, at sufficiently high energies, cosmic-ray protons interact with the photons in the Cosmic Microwave background. In that process other particles are produced, such as neutrinos, that carry an appreciable fraction of the original energy. The flux of energetic cosmic rays decreases as a result. Since neutrinos interact exclusively via the weak interaction they can propagate over cosmological distances. Neutrinos are also expected to be produced at the sources of cosmic rays. Either way, neutrinos point back to the original cosmic rays. For this reason we have set out to detect UHE neutrinos, which requires very large and efficient detectors. One particular approach which we describe here is to determine UHE particle fluxes through the detection of coherent radio waves which are emitted when an UHE particle interacts with the lunar surface.

\section{Cherenkov radiation}

In a dielectric, the propagation speed of electromagnetic waves is reduced by a factor equal to the index of refraction. A charge moving at light-speed through such a dielectric will emit Cherenkov radiation, a process which is similar to the emission of sound waves (the sonic boom) when an aeroplane exceeds the velocity of sound. An UHE particle, neutrino as well as cosmic ray, with an energy of $10^{18} \mathrm{eV}$ that interacts with the surface layer of the Moon (the regolith) will generate a cascade of particles, which may contain $10^{8}$ particles (mostly electrons and positrons) all moving with velocities close to the speed of light in vacuum. The number of particles scales linearly with the initial energy. Due to processes such as Compton scattering and positron collisions off electrons bound to the atoms of the regolith, a net excess of electrons is formed in the shower front. This excess is typically equal to about a third of the number of particles in the shower. Because of the large energies involved, all particles stay close together and can be envisioned as a large charged cloud moving at a velocity close to the speed of light, with a typical dimension of $10 \mathrm{~cm}$. This moving charge will thus emit Cherenkov radiation. At wavelengths exceeding $10 \mathrm{~cm}$ (frequency less than $3 \mathrm{GHz}$ ) the individual charges cannot be distinguished and the emitted radiation is coherent. The emitted power is proportional to the square of the number of particles in the cascade. Evidence for coherent radiation from the charge excess [4] has recently been observed [5] as radio emission from cosmic-rayinduced air showers at the Pierre Auger Observatory in the Argentinian pampas. At sufficiently high energies, the emitted pulse on the Moon is strong enough to be detectable with radio telescopes on Earth. With a visible area of $10^{7} \mathrm{~km}^{2}$, the Moon can be used as a highly efficient detector for UHE particles since the lunar surface layer, the regolith, consists of dust and small rocks and is very transparent to radio waves. The regolith has been created in a constant bombardment by meteorites. In some places it has a thickness of several kilometres. A number of experiments have been performed looking for radiation at frequencies around $2 \mathrm{GHz}$ where the intensity is expected to reach its maximum [6]. At these frequencies the
4 the moon during a lunar eclipse (c) iStockPhoto 


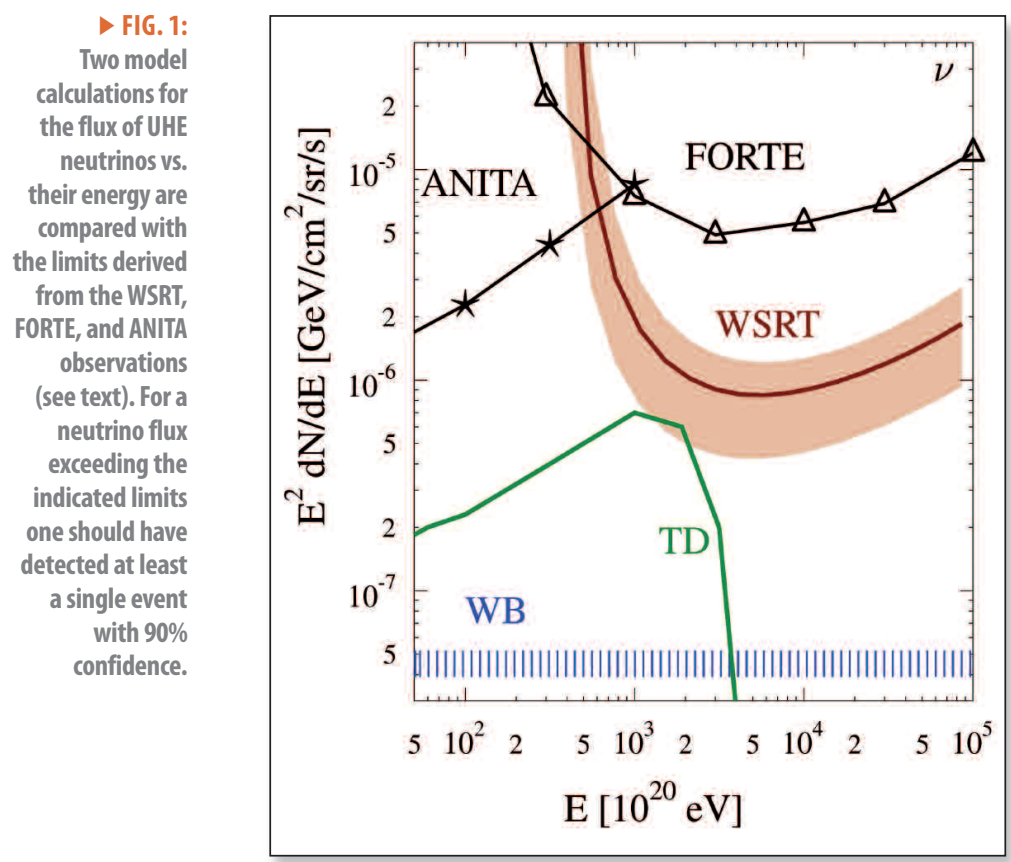

- emitted radiation is sharply focused at the Cherenkov angle since the distance over which the shower travels, about $3 \mathrm{~m}$, is much larger than the wavelength, $15 \mathrm{~cm}$ at $2 \mathrm{GHz}$.

At lower frequencies the angular spread of the emitted radiation increases to a maximum at frequencies of $100-200 \mathrm{MHz}$ where the wavelength is comparable to the longitudinal extent of the shower. At even lower frequencies there is only a marginal increase in angular spread while the intensity of the emitted radiation drops linearly with frequency. The increase of the angular spread of the radio emission is important for two reasons. First, the radiation created will be detectable on Earth for many more angles of incidence. Second, for a shower parallel to the lunar surface the Cherenkov emission angle is the same as the angle of total internal reflection at the surface. Thus, only the UHE particles impinging at the rim of the Moon in the direction towards the Earth will emit a detectable signal at high frequencies. At lower frequencies, the particles impinging anywhere in any direction on the lunar surface may emit detectable signals. Because of the large attenuation length, radio waves created at large depths (as much as $500 \mathrm{~m}$ is very well possible) may also result in a detectable signal at Earth, which makes for a very large detection volume. These effects combined result in a detection efficiency that increases with about the third power of the wavelength [7].

Cosmic rays interact directly at the lunar surface because of their rather large interaction cross section. They convert their entire energy into a particle cascade, called the hadronic shower, in the uppermost layer of the regolith. UHE neutrinos, on the other hand, may traverse many kilometres of rock before they interact, and in the interaction about
$20 \%$ of their energy is converted into a hadronic shower, while the remaining energy is carried off by a single lepton.

In both cases - cosmic rays and UHE neutrinos coherent Cherenkov radiation will be emitted by the many particles in the hadronic shower. In this article we will limit ourselves to neutrino detection.

\section{Observations}

We have observed the Moon with the Westerbork Synthesis Radio Telescope (WSRT) in the frequency range of $115-180 \mathrm{MHz}$ with full polarization sensitivity. These observations have produced the most stringent flux limit yet at the highest energies [8]. The observations were made using eleven equally-spaced WSRT parabolic antennas of $25 \mathrm{~m}$ diameter. Our particular set-up created two simultaneous beams aimed at different sides of the Moon. This allowed us to implement an anti-coincidence condition since a lunar Cherenkov pulse should be visible in only one of them.

To analyse the data from the WSRT observations, we did not follow the usual procedure in astronomy where one determines the intensity at a particular well defined frequency, which requires signal integration over appreciable time-scales. In the present case we are instead interested in pulses that have a very short, nanosecond, structure which requires a large bandwidth in frequency. In this wide band, terrestrial communication frequencies need to be filtered out. A further complication is the dispersion of radio signals by the ionosphere of the Earth. In the analysis, we corrected for this frequency-dependent delay by using the ionospheric information obtained from GPS data.

In the off-line analysis the data are 'cleaned' further by eliminating pulses that are very wide, and pulses that appear in both beams in the same time trace. By simulations, we found that there was no pulse from the Moon with a strength exceeding 120 times that of the Galactic noise level in 125 nanoseconds. This non-detection in a time span of $47.6 \mathrm{~h}$ converts into a limit on the flux of neutrinos, where attenuation of the signal in the Moon, transmission at the lunar surface, and angle with respect to the direction of the neutrino have to be taken into account. The resulting $90 \%$ confidence flux limit is shown in figure 1 by the curve labeled WSRT. The band around the curve shows the possible error of the determined flux limit, mostly due to uncertainties in the attenuation of radio waves in the regolith.

To place the observations in context, they are compared with those by the FORTE satellite [9], observing the Greenland ice cap for radio flashes coming from neutrino interactions in the ice cap, and from the ANITA balloon mission where the Antarctic ice cap is 
searched for radio flashes [10]. The Figure gives also two model predictions. Waxman and Bahcall (WB) derived an estimate of the flux of neutrinos based on the observed flux of UHE cosmic rays assuming a generic model for neutrino production at the production sites of the cosmic rays [11]. In Top-Down (TD) models UHE neutrinos are created from the decay of super massive relics $\left(\mathrm{M}_{\mathrm{x}}=10^{24} \mathrm{eV}\right.$ is used in the figure) formed at the time of the Big Bang. The new data from Westerbork allows for setting much a tighter limit on the flux of UHE neutrinos which is just touching the predicted flux of a TD model.

\section{Recent developments}

LOFAR (Low Frequency Array) is a new-concept radio telescope presently being completed in The Netherlands and surrounding countries. LOFAR combines thousands of simple wire dipole antennas. In software-manipulation, the relative phasing of the antennas is tuned such that the array can be pointed towards any direction in the sky. Because such software pointing does not require any physical antenna movement, multiple beams may be observed simultaneously. The large collecting area benefits the signal-to-noise ratio. The multi-beam option allows for the formation of a sufficient number of beams to cover the entire lunar surface. The long baselines make the position resolution on the Moon very precise which improves the sensitivity to localized pulses as generated by neutrino impacts.

With the much higher sensitivity of LOFAR weaker pulses and hence neutrinos at lower energies can be detected than was possible with the WSRT. In Figure 2, we give the flux limit that is reached in a week of observation with LOFAR. This limit implies that we will detect a dozen events if the flux of neutrinos is of the magnitude estimated by Waxman and Bahcall. This is very exciting as it would constitute the first detection of UHE neutrinos. In the Figure we also give an estimate for the neutrino flux due to the GZK effect but this is rather model-dependent [12].

\section{Outlook}

Already advanced plans exist for a successor to LOFAR, called the Square Kilometre Array (SKA). The SKA will be built using the same aperture-array concept as LOFAR where many simple antennas are coupled through software to effectively simulate a gigantic single-dish antenna. SKA will have a collecting area of about $1 \mathrm{~km}^{2}$ which is much larger than that of LOFAR. A larger collecting area allows for the detection of fainter objects. For lunar pulse observations it is also important that the full frequency range of interest is covered. It ranges from $100 \mathrm{MHz}$, corresponding to a wavelength comparable to the longitudinal extent of the shower, up to a few $\mathrm{GHz}$, corresponding to a wavelength comparable to the size of the moving charge cloud.

Such increases in collecting area and receiver bandwidth allow us to detect neutrinos created in the interaction of UHE protons with the Cosmic Microwave Background as well as the signals caused by the impacts of cosmic rays on the lunar surface with energies as low as $10^{20} \mathrm{eV}$. The sensitivity for cosmic ray detection will be such that we will collect the same number of counts in a single week as in a few years of operation of the Pierre Auger Observatory.

\section{References}

[1] J. Abraham et al. [The Pierre Auger Collaboration], Phys. Lett. B 685,239 (2010).

[2] For a review of possible sources of UHE cosmic rays see R.J. Protheroe and R.W. Clay, PASA 21 (2004) p1; www.publish.csiro.au/paper/AS03047.htm

[3] P. Abreu et al. [The Pierre Auger Collaboration], Astroparticle Physics 34, 314 (2010).

[4] O. Scholten, K.D. de Vries, K. Werner, Proceedings ARENA 2010; arXiv:1010.5268.

[5] H. Schoorlemmer et al. [The Pierre Auger Collaboration], Proceedings of ARENA 2010; doi:10.1016/j.nima.2010.11.145

[6] For a general overview see "Moon may reveal elusive cosmic neutrinos" by Rachel Courtland, New Scientist, www.newscientist.com/article/dn17561-moon-used-as-giant-particle-d etector.html

[7] O. Scholten et al., Astropart. Phys. 26 (2006) 219.

[8] O. Scholten et al., Phys. Rev. Lett. 103 (2009) 191301; S. Buitink et al., Astron. \& Astroph. 521 (2010) A47.

[9] H. Lethinen et al., Phys. Rev. D 69, 013008 (2004).

[10] P. Gorham et al. (ANITA collaboration), Phys. Rev. D 82, 022004 (2010); erratum arXiv:1003.2961 .

[11] E. Waxman and J.N. Bahcall, Phys. Rev. D 59, 023002 (1999)

[12] V. Barger, P. Huber, D. Marfatia, Phys. Lett. B 642, 333 (2006) doi: 10.1016/j.physletb.2006.09.067; arXiv:hep$\mathrm{ph} / 0606311 \mathrm{v} 1$

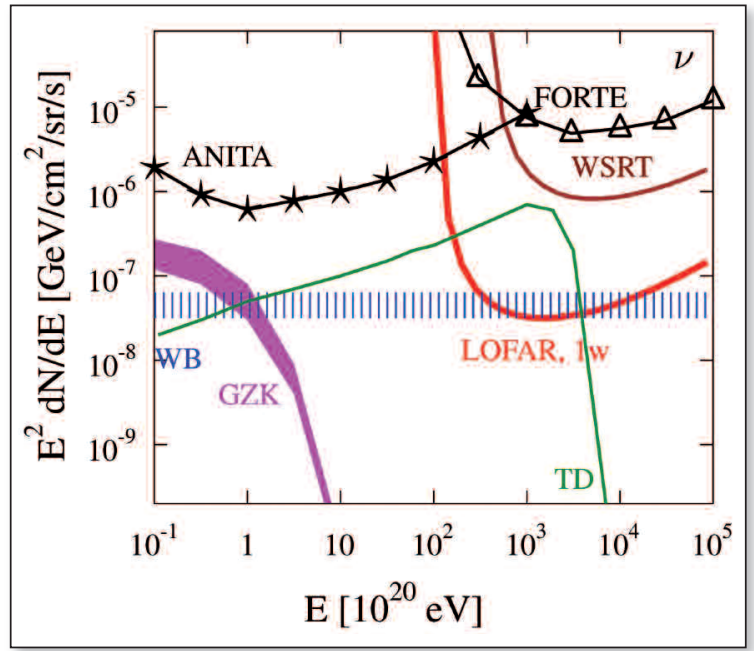

4 FIG. 2:

The predicted sensitivity for LOFAR is compared with the results obtained from the NuMoon observations with WSRT. 